Article

\title{
Polypharmacy and Depressive Symptoms in U.S.-Born Mexican American Older Adults
}

\author{
Shervin Assari ${ }^{1, *(\mathbb{D}, \text { Cheryl Wisseh }}{ }^{2}$, Mohammed Saqib ${ }^{3}$, Hamid Helmi ${ }^{4}$ and \\ Mohsen Bazargan 1,5 \\ 1 Department of Family Medicine, Charles R Drew University of Medicine and Science, Los Angeles, \\ CA 90059, USA; mohsenbazargan@cdrewu.edu \\ 2 Department of Pharmacy Practice, West Coast University School of Pharmacy, Los Angeles, CA 91606, \\ USA; cWisseh@westcoastuniversity.edu \\ 3 Department of Health Behavior and Health Education, University of Michigan, Ann Arbor, MI 48109, \\ USA; saqimoha@umich.edu \\ 4 School of Medicine, Wayne State University, Detroit, MI 48202, USA; hhelmi@umich.edu \\ 5 Department of Family Medicine, University of California, Los Angeles (UCLA), Los Angeles, CA 90095, USA \\ * Correspondence: assari@umich.edu; Tel.: +1-(734)-232-0445; Fax: +1-734-615-8739
}

Received: 13 September 2019; Accepted: 30 October 2019; Published: 1 November 2019

check for updates

\begin{abstract}
Background: Although some studies have suggested a link between polypharmacy and poor mental health, less is known about the association between polypharmacy and depressive symptomology among U.S.-born older Mexican Americans. Aim: This study aimed to test the association between polypharmacy and depressive symptoms in U.S.-born older Latino Americans. Materials and methods: Data came from the Sacramento Area Latino Study on Aging (SALSA 2008). A total of 691 U.S.-born older (age $>=65$ ) Mexican Americans entered this analysis. Polypharmacy was the independent variable. Level of depressive symptoms was the outcome. Age, gender, socioeconomic status (education, income, and employment), retirement status, health (chronic medical conditions, self-rated health, and activities of daily living), language, acculturation, and smoking were the covariates. A linear regression model was used to analyze the data. Results: We found a positive association between polypharmacy and depressive symptoms, which was above and beyond demographic factors, socioeconomic status, physical health, health behaviors, language, acculturation, and health insurance. Conclusion: Polypharmacy is linked to depressive symptoms in U.S.-born older Mexican Americans. More research is needed to test the effects of reducing inappropriate polypharmacy on mental well-being of first and second generation older Mexican Americans. There is also a need to study the role of drug-drug interaction in explaining the observed link between polypharmacy and depressive symptoms.
\end{abstract}

Keywords: Mexican Americans; ethnicity; Hispanics; Latino; ethnic groups; polypharmacy; depressive symptoms

\section{Introduction}

Polypharmacy is associated with undesired physical health outcomes such as death [1]. Given the serious consequences and the social and health care costs involved, epidemiologists have shown an increasing interest in understanding mental health correlates of polypharmacy across diverse populations [2]. This is particularly important because polypharmacy has unequal correlates across ethnic groups [3]. This means one factor may correlate with polypharmacy in one but not another ethnic group [3].

Thus, there is a need to conduct epidemiological studies that investigate mental health correlates of polypharmacy in ethnic groups that are rarely studied [4,5]. Although some studies suggest 
polypharmacy is associated with depressive symptoms and psychological distress [6,7], not much is known about the link between polypharmacy and poor mental health in Mexican American older adults.

To test the independent association between polypharmacy and mental health, there is a need to control for a wide range of third varibles that can potentially confound such an association. The first group of potential confounders are demographic factors such as age and gender [7-10]. It has been demonstrated that polypharmacy is more common in women, and older individuals, who have a higher number of chronic diseases and frequent contact with the health care system [7-10]. At the same time, depressive symptoms are also more common in women than men [11-15]. Thus, there is a need to control for age and gender when we test the link between polypharmacy and depressive symptoms in a particular population group.

Socioeconomic status (SES) is also associated with both polypharmacy [4,5] and mental health [16, 17]. Polypharmacy is more common in people with low educational attainment and income [4,5]. This might be simply because illnesses may emerge as a result of high stress and adversities, and poor health behaviors, which may arise as a result of low socioeconomic status [4,5]. Individuals with low income and low educational attainment also experience more depressive symptoms $[16,17]$. Thus, any study on the potential link between polypharmacy and depressive symptoms should control for SES indicators such as education, income, and employment $[3,6,7,18]$.

Physical health, particularly self-rated health (SRH), chronic medical conditions (CMC), and activities of daily living (ADL), are associated with polypharmacy and depression [19-21]. Polypharmacy is also a consequence of being diagnosed with multiple conditions $[6,7,10,18]$. This means that these health indicators are strong determinants and correlates of polypharmacy $[6,7,10,18]$. Poor physical health (CMC, SRH, and ADL) and depressive symptomatology also have bidirectional associations [19-21]. As a result, there is a need to control for physical health (e.g., CMC, SRH, and ADL) in the studies that wish to understand the association between polypharmacy and depressive symptoms $[6,7,10,18]$.

What we know about the interplay between SES, physical health, mental health, and polypharmacy in ethnic minorities is very limited [3]. Across various ethnic minorities in the U.S., less is known about Latinos/Hispanics than African Americans [3,18]. Some studies have suggested different pattern of associations and sometimes inconsistencies in these associations in ethnic minorities [3,18]. These inconsistencies may be due to the role of context and culture in shaping correlates of health across settings [22-26].

As a result, there is a need to explore how polypharmacy and depressive symptoms are linked in Latino older Americans. There is also a need to study how these links are robust after adjusting for demographic factors, SES, physical health, healthcare access, language, and acculturation. As Latino Americans are composed of diverse and heterogenous groups that vary by country of origin, nativity, legal status, and immigration status, there is a need for studies that explore the link between these factors in each specific group of Latino Americans. To fill such a gap, the current study investigates the association between polypharmacy and depressive symptoms specifically in older Mexican Americans who were born in U.S. We hypothesized that polypharmacy is associated with more depressive symptoms in a national sample of U.S.-born older Mexican Americans, and this association would remain significant after controlling for demographic factors, SES, physical health, healthcare access, acculturation, and language.

\section{Materials and Methods}

\subsection{Design and Setting}

The Sacramento Area Latino Study on Aging (SALSA) is a study of older Mexican Americans in the U.S. [27,28]. Sampling, design, measures, and rationale are extensively described previously [27-33]. To describe the study briefly, SALSA is a representative study of Hispanics in the Sacramento 
County and neighboring counties. The data collected comprises rich information on demographics, SES, medication use, physical health, mental health, healthcare access, and cultural factors.

\subsection{Ethics}

All participants signed a written consent form. The SALSA protocol was reviewed and approved by the Institutional Review Boards of the University of California (UC), Davis and University of Michigan (UM), Ann Arbor.

\subsection{Sample and Sampling}

Participants were sampled from counties with proportional densities of Hispanics of at least $5 \%$, based on U.S. Census information 1990. Participants were eligible if they were: (1) self-identified as Latino or Hispanic; (2) Spanish or English speaking; (3) age 65+ years; and (4) U.S. born. The exclusion criterion was living in an institutionalized setting. Both Spanish and English speakers were enrolled. Approximately $22 \%$ of the total eligible population of Sacramento County, CA, was recruited into the SALSA study and a total number of 691 individuals entered this study.

\subsection{Dependent Variable (Outcome)}

Depressive symptoms were the outcome. Depressive symptoms were measured using a self-reported measure called Center for Epidemiologic Studies Depression Scale (CES-D). The CES-D includes 20 items that query the participants regarding their depressive symptoms over the past 4 weeks. Item responses range from 0 to 3 . The total score ranges from 0 to 60 . The CES-D generated a continuous score, with a higher score indicative of more symptoms $[28,33,34]$.

\subsection{Independent Variable (Predictor)}

Polypharmacy was the independent variable. This study defined polypharmacy as use of $5+$ medications in a single day. This definition was based on a systemic review of definitions of polypharmacy across studies. Although there is no consensus, this is probably the most common definition of polypharmacy used by researchers [1]. In this study, polypharmacy was calculated based on a comprehensive evaluation of all medications that the individual was taking.

\subsection{Covariates (Confounders)}

This study also included age, gender, SES (education, income, and employment), retirement status, health insurance, self-rated health (SRH), number of chronic medical conditions (CMCs), smoking, language, healthcare access, ethnic identity, and acculturation as covariates. Age was a continuous measure and gender was a dichotomous measure. For gender, male was 1 and female was 0 . Participants were asked if they were retired. Health insurance was self-reported, regardless of its type. Three SES indicators were measured as self-reported data. Education was defined as years of formal education and schooling. Household annual income was also self-reported. Education and income were continuous measures. Employment was a dichotomous variable: $1=$ employed, $0=$ not in the labor market or unemployed. Smoking was also a dichotomous variable where 1 indicated a current smoker, and 0 indicated never smoked or a past history of smoking.

Number of CMCs was measured using self-reported data. Participants reported the presence of cardiovascular disease (e.g., myocardial infarction, angina pectoris, stroke, heart failure, intermittent claudication, atrial fibrillation, deep vein thrombosis, and heart/coronary catheterization), stroke, diabetes, hypertension, kidney disease, pneumonia, gall bladder problems, and liver disease. Participants were asked if any physician had ever diagnosed them with any of the above conditions. Number of CMCs was conceptualized as a continuous measure, with a higher score indicating multimorbidity. 
Self-Rated Health (SRH) was the conventional single item, ranging from 1 to 5, with a higher score indicating worse health. The item read as "Would you say, in general, your health is: 1 Excellent, 2 Very good, 3 Good, 4 Fair, and 5 Poor?". We treated SRH as a continuous measure.

Activities of daily living (ADLs) were measured applying a standard Likert scale [35,36]. Participants were asked about their ability to perform 13 common ADLs. Responses ranged from 0 to 2, 0 for no need, 1 for needed some help, and 2 needed help all the time. ADL was operationalized as a continuous measure ranging from 0 to 26 , with a higher score yielding higher disability $[35,36]$.

Acculturation was measured using the Geriatric Acculturation Ratings Scale for Mexican Americans (G-ARSMA). The G-ARSMA, as developed by Cuellar et al., consists of 19 items. Items assessed English and Spanish media use, spoken language, maintenance of childhood friendships, building new friendships, frequency of contact with Latin America, and cultural and ethnic dietary practices. Highest scores represent Anglo-oriented participants, middle sores represent bicultural participants, and the lowest score represents least acculturated participants (Mexican-oriented) [37-39].

Self-reported language proficiency was the other covariate. Participants rated how well they spoke English and Spanish on a four-point scale: 0-not at all, 1-a little, 2-well, 3-very well. Use of English versus Spanish language was coded if Spanish or English was spoken "very often", "almost always", "not very often", or "not at all". Latino identity was a single item with 4 item responses, with a higher score indicating stronger ethnic ties.

\subsection{Statistics}

We used SPSS 23.0 for data analysis. Frequencies, means, and standard deviations (SDs) of our variables were reported to describe the sample. For bivariate analysis, we ran the Spearman correlation test. For multivariable analysis, we ran a hierarchical multivariable linear regression model. This model had polypharmacy as the independent variable, and depressive symptoms as the outcome. Specifically, a 6-step hierarchical regression model was applied. Demographics, SES, health, cultural, and health behaviors were conceptualized as blocks of covariates. Model 1 only included 1 demographic factor. Model 2 included SES indicators in addition to the variable in Model 1. Model 3 included cultural factors in addition to the variables in Model 1 and Model 2. Model 4 included health variables in addition to the variables in Models 1-3. Model 5 added smoking in addition to the variables in Models 1-4. Model 6 added polypharmacy in addition to the variables in Models 1-5. Beta coefficient (B), 95\% confidence interval (95\% CI), Standard Error (SE), and $p$-value were reported.

\section{Results}

\subsection{Univariate Analysis}

Table 1 shows a summary of descriptive statistics for our sample. All individuals were at least 65 years old. On average, our sample was 72 years of age. In our sample, 55.2\% were women, $85.2 \%$ were retired, and only $0.9 \%$ did not have health insurance. Only $12.7 \%$ of our participants were working at the time of survey. From all participants, $23.2 \%$ exhibited polypharmacy. 
Table 1. Descriptive statistics.

\begin{tabular}{|c|c|c|}
\hline & $\mathbf{n}$ & $\%$ \\
\hline \multicolumn{3}{|l|}{ Gender } \\
\hline Women & 369 & 55.2 \\
\hline Men & 300 & 44.8 \\
\hline \multicolumn{3}{|l|}{ Employment } \\
\hline No & 583 & 87.3 \\
\hline Yes & 85 & 12.7 \\
\hline \multicolumn{3}{|l|}{ Retirement } \\
\hline No & 99 & 14.8 \\
\hline Yes & 568 & 85.2 \\
\hline \multicolumn{3}{|l|}{ Insurance } \\
\hline No & 6 & 0.9 \\
\hline Yes & 682 & 99.1 \\
\hline \multicolumn{3}{|l|}{ Smoking (Current) } \\
\hline No & 616 & 89.4 \\
\hline Yes & 73 & 10.6 \\
\hline \multicolumn{3}{|l|}{ Polypharmacy } \\
\hline No & 531 & 76.8 \\
\hline \multirow[t]{2}{*}{ Yes } & 160 & 23.2 \\
\hline & Mean & SD \\
\hline Age (Years) & 72.10 & 5.60 \\
\hline Education (Years) & 2.70 & 1.53 \\
\hline Household Income & 9.10 & 5.03 \\
\hline Spanish Language (1-4) & 2.06 & 0.91 \\
\hline Acculturation & 44.87 & 10.03 \\
\hline Latino Identity & 2.89 & 0.52 \\
\hline Self-Rated Health [SRH] (1-5) & 3.13 & 1.09 \\
\hline $\begin{array}{l}\text { Activities of Daily Living [ADL] } \\
\text { (n) }\end{array}$ & 0.76 & 2.73 \\
\hline $\begin{array}{l}\text { Chronic Medical Conditions } \\
\text { [CMC] (n) }\end{array}$ & 1.84 & 1.61 \\
\hline Depressive Symptom & 8.33 & 9.62 \\
\hline
\end{tabular}

\subsection{Bivariate Analysis}

Table 2 shows the results of bivariate correlations in our sample. In bivariate analysis, the level of depressive symptoms was associated with gender, education, income, employment, Spanish language proficiency, acculturation, SRH, ADL, CMC, and polypharmacy. Other variables did not correlate with the level of depressive symptoms in univariate analysis. 
Table 2. Bivariate correlations among participants.

\begin{tabular}{|c|c|c|c|c|c|c|c|c|c|c|c|c|c|c|c|c|}
\hline & 1 & 2 & 3 & 4 & 5 & 6 & 7 & 8 & 9 & 10 & 11 & 12 & 13 & 14 & 15 & 16 \\
\hline 1 Gender (Men) & 1.00 & $\underset{* *}{-0.10}$ & $0.34^{* *}$ & $0.14 * *$ & 0.07 & -0.04 & 0.02 & -0.07 & 0.06 & $\underset{* *}{-0.11}$ & -0.06 & -0.05 & 0.03 & $0.14^{* *}$ & -0.06 & $\underset{* *}{-0.17}$ \\
\hline 2 Age (Years) & & 1.00 & $\begin{array}{c}-0.27 \\
* *\end{array}$ & $\underset{* *}{-0.26}$ & $\begin{array}{c}-0.18 \\
* *\end{array}$ & $0.15^{* *}$ & 0.04 & $0.12^{* *}$ & $-0.09 *$ & $\begin{array}{c}-0.10 \\
* *\end{array}$ & $0.08 *$ & $0.12^{* *}$ & 0.06 & $-0.09 *$ & 0.03 & $0.10 *$ \\
\hline $\begin{array}{l}3 \text { Household } \\
\text { Income }\end{array}$ & & & 1.00 & $0.50 * *$ & $0.19 * *$ & -0.15 & -0.01 & $\underset{* *}{-0.24}$ & $0.33^{* *}$ & -0.04 & $\underset{* *}{-0.32}$ & $\underset{* *}{-0.18}$ & $-0.09 *$ & -0.02 & $-0.09 *$ & $\underset{* *}{-0.33}$ \\
\hline 4 Education (Years) & & & & 1.00 & $0.19 * *$ & $\underset{* *}{-0.11}$ & -0.01 & $\underset{* *}{-0.41}$ & $0.52 * *$ & -0.02 & $\underset{* *}{-0.33}$ & $\underset{* *}{-0.14}$ & $-\underset{* *}{-0.13}$ & -0.04 & -0.10 * & $\underset{* *}{-0.29}$ \\
\hline 5 Employed & & & & & 1.00 & $\underset{* *}{-0.73}$ & $\underset{* *}{-0.11}$ & -0.07 & $0.12 * *$ & -0.05 & $\underset{* *}{-0.14}$ & -0.05 & $-0.09 *$ & -0.04 & $\underset{* *}{-0.11}$ & $\underset{* *}{-0.13}$ \\
\hline $\begin{array}{c}6 \text { Retired } \\
7 \text { Health insurance }\end{array}$ & & & & & & 1.00 & $\begin{array}{l}0.05 \\
1.00\end{array}$ & $\begin{array}{l}0.04 \\
0.04\end{array}$ & $\begin{array}{l}-0.05 \\
0.00\end{array}$ & $\begin{array}{c}0.01 \\
-0.02\end{array}$ & $\begin{array}{l}0.05 \\
0.06\end{array}$ & $\begin{array}{c}0.05 \\
-0.01\end{array}$ & $\begin{array}{l}0.07 \\
0.02\end{array}$ & $\begin{array}{l}0.03 \\
0.03\end{array}$ & $\begin{array}{c}0.07 \\
-0.02\end{array}$ & $\begin{array}{l}0.10^{* *} \\
0.06\end{array}$ \\
\hline $\begin{array}{c}8 \text { Spanish } \\
\text { Language }(1-4)\end{array}$ & & & & & & & & 1.00 & $\begin{array}{c}-0.75 \\
* *\end{array}$ & 0.05 & $0.16^{* *}$ & $0.19^{* *}$ & 0.00 & 0.03 & 0.00 & $0.11^{* *}$ \\
\hline 9 Acculturation & & & & & & & & & 1.00 & -0.15 & $\underset{* *}{-0.20}$ & $\underset{* *}{-0.20}$ & -0.01 & 0.00 & -0.05 & -0.22 \\
\hline 10 Latino Identity & & & & & & & & & & 1.00 & 0.02 & -0.02 & 0.02 & -0.02 & 0.02 & 0.03 \\
\hline 11 SRH (1-5) & & & & & & & & & & & 1.00 & $0.25 * *$ & $0.32 * *$ & 0.02 & $0.30 * *$ & $0.37^{* *}$ \\
\hline $12 \mathrm{ADL}$ & & & & & & & & & & & & 1.00 & $0.22 * *$ & 0.05 & $0.20 * *$ & $0.21 * *$ \\
\hline 13 CMC (n) & & & & & & & & & & & & & 1.00 & 0.01 & $0.45^{* *}$ & $0.22 * *$ \\
\hline 15 Polypharmacy & & & & & & & & & & & & & & & 1.00 & $0.21^{* *}$ \\
\hline $\begin{array}{l}16 \text { Depressive } \\
\text { Symptoms }\end{array}$ & & & & & & & & & & & & & & & & 1.00 \\
\hline
\end{tabular}




\subsection{Multivariable Model}

Table 3 shows the results of the six-step hierarchical regression model. Model 1 only included the demographic factor. Model 2 included SES indicators in addition to the variable in Model 1. Model 3 included cultural factors in addition to the variables in Models 1 and 2. Model 4 included health variables in addition to the variables in Models 1-3. Model 5 added smoking in addition to the variables in Models 1-4. Model 6 added polypharmacy in addition to the variables in Models 1-5.

Table 3. Summary of hierarchical regression model on social, behavioral, cultural, and health determinants of depressive symptoms.

\begin{tabular}{|c|c|c|c|c|c|c|}
\hline & B & $\mathrm{SE}$ & Beta & \multicolumn{2}{|c|}{$95 \% \mathrm{CI}$ for B } & $P$ \\
\hline \multicolumn{7}{|l|}{ Model 1} \\
\hline Gender (Men) & -1.90 & 0.80 & -0.12 & -3.47 & -0.33 & 0.018 \\
\hline Age (Years) & 0.10 & 0.08 & 0.06 & -0.06 & 0.26 & 0.211 \\
\hline Intercept & 0.49 & 5.72 & & -10.75 & 11.74 & 0.931 \\
\hline \multicolumn{7}{|l|}{ Model 2} \\
\hline Gender (Men) & -0.59 & 0.79 & -0.04 & -2.15 & 0.97 & 0.458 \\
\hline Age (Years) & -0.03 & 0.08 & -0.02 & -0.18 & 0.13 & 0.715 \\
\hline Household Income & -1.27 & 0.30 & 0.25 & -1.85 & -0.68 & 0.000 \\
\hline Education (Years) & -0.26 & 0.09 & 0.16 & 0.44 & 0.09 & 0.004 \\
\hline Employed & -0.23 & 1.80 & -0.01 & 3.78 & 3.31 & 0.898 \\
\hline Retired & -0.78 & 1.71 & -0.04 & -4.14 & 2.59 & 0.651 \\
\hline Health insurance & 3.86 & 4.28 & 0.04 & -4.55 & 12.28 & 0.368 \\
\hline Intercept & 12.41 & 7.38 & & -2.09 & 26.92 & 0.093 \\
\hline \multicolumn{7}{|l|}{ Model 3} \\
\hline Gender (Men) & -0.69 & 0.80 & -0.04 & -2.27 & 0.88 & 0.387 \\
\hline Age (Years) & -0.02 & 0.08 & -0.01 & -0.17 & 0.14 & 0.834 \\
\hline Household Income & -1.23 & 0.30 & -0.24 & -1.83 & -0.64 & 0.000 \\
\hline Education (Years) & -0.23 & 0.10 & -0.14 & -0.42 & -0.03 & 0.021 \\
\hline Employed & 0.03 & 1.82 & 0.00 & -3.56 & 3.61 & 0.988 \\
\hline Retired & -0.61 & 1.72 & -0.03 & -3.99 & 2.77 & 0.722 \\
\hline Health insurance & 4.05 & 4.29 & 0.05 & -4.39 & 12.48 & 0.346 \\
\hline $\begin{array}{c}\text { Spanish Language } \\
\qquad(1-4)\end{array}$ & -0.49 & 0.59 & -0.06 & -1.66 & 0.67 & 0.407 \\
\hline Acculturation & -0.08 & 0.07 & -0.10 & -0.21 & 0.05 & 0.201 \\
\hline Latino Identity & -0.14 & 0.74 & -0.01 & -1.59 & 1.30 & 0.845 \\
\hline Intercept & 16.08 & 8.62 & & -0.86 & 33.02 & 0.063 \\
\hline \multicolumn{7}{|l|}{ Model 4} \\
\hline Gender (Men) & -1.41 & 0.78 & -0.09 & -2.94 & 0.11 & 0.070 \\
\hline Age (Years) & 0.01 & 0.08 & 0.01 & -0.14 & 0.16 & 0.904 \\
\hline Household Income & -0.91 & 0.29 & -0.18 & -1.49 & -0.33 & 0.002 \\
\hline Education (Years) & -0.12 & 0.10 & -0.08 & -0.31 & 0.06 & 0.196 \\
\hline Employed & 0.11 & 1.75 & 0.00 & -3.33 & 3.54 & 0.952 \\
\hline Retired & -1.03 & 1.65 & -0.05 & -4.27 & 2.22 & 0.534 \\
\hline Health insurance & 4.41 & 4.14 & 0.05 & -3.73 & 12.55 & 0.288 \\
\hline $\begin{array}{c}\text { Spanish Language } \\
(1-4)\end{array}$ & -0.65 & 0.57 & -0.07 & -1.77 & 0.47 & 0.256 \\
\hline Acculturation & -0.10 & 0.06 & -0.11 & -0.22 & 0.03 & 0.131 \\
\hline Latino Identity & 0.03 & 0.71 & 0.00 & -1.37 & 1.42 & 0.970 \\
\hline SRH (1-5) & 1.56 & 0.39 & 0.22 & 0.79 & 2.34 & 0.000 \\
\hline $\mathrm{ADL}$ & 0.73 & 0.36 & 0.10 & 0.02 & 1.44 & 0.043 \\
\hline CMC (n) & 0.67 & 0.27 & 0.12 & 0.15 & 1.19 & 0.012 \\
\hline Intercept & 7.13 & 8.43 & & -9.44 & 23.70 & 0.398 \\
\hline
\end{tabular}


Table 3. Cont.

\begin{tabular}{|c|c|c|c|c|c|c|}
\hline & B & $\mathrm{SE}$ & Beta & \multicolumn{2}{|c|}{$95 \% C I$ for $B$} & $P$ \\
\hline \multicolumn{7}{|l|}{ Model 5} \\
\hline Gender (Men) & -1.48 & 0.79 & -0.09 & -3.03 & 0.08 & 0.062 \\
\hline Age (Years) & 0.01 & 0.08 & 0.01 & -0.14 & 0.17 & 0.856 \\
\hline Household Income & -0.89 & 0.30 & -0.17 & -1.48 & -0.31 & 0.003 \\
\hline Education (Years) & -0.12 & 0.10 & -0.08 & -0.31 & 0.07 & 0.212 \\
\hline Employed & 0.10 & 1.75 & 0.00 & -3.33 & 3.54 & 0.952 \\
\hline Retired & -1.05 & 1.65 & -0.05 & -4.30 & 2.20 & 0.525 \\
\hline Health insurance & 4.37 & 4.15 & 0.05 & -3.78 & 12.52 & 0.293 \\
\hline $\begin{array}{c}\text { Spanish Language } \\
\qquad(1-4)\end{array}$ & -0.66 & 0.57 & -0.08 & -1.78 & 0.46 & 0.247 \\
\hline Acculturation & -0.10 & 0.06 & -0.11 & -0.22 & 0.03 & 0.126 \\
\hline Latino Identity & 0.04 & 0.71 & 0.00 & -1.36 & 1.44 & 0.955 \\
\hline SRH (1-5) & 1.57 & 0.39 & 0.22 & 0.80 & 2.35 & 0.000 \\
\hline ADL & 0.73 & 0.36 & 0.10 & 0.02 & 1.44 & 0.044 \\
\hline CMC (n) & 0.66 & 0.27 & 0.12 & 0.14 & 1.19 & 0.014 \\
\hline Smoking (Current) & 0.54 & 1.16 & 0.02 & -1.75 & 2.83 & 0.643 \\
\hline $\begin{array}{l}\text { Intercept } \\
\text { Model } 6\end{array}$ & 6.79 & 8.47 & & -9.86 & 23.45 & 0.423 \\
\hline Gender (Men) & -1.43 & 0.79 & -0.09 & -2.97 & 0.12 & 0.070 \\
\hline Age (Years) & 0.01 & 0.08 & 0.00 & -0.15 & 0.16 & 0.938 \\
\hline Household Income & -0.94 & 0.29 & -0.18 & -1.52 & -0.36 & 0.002 \\
\hline Education (Years) & -0.13 & 0.10 & -0.08 & -0.32 & 0.06 & 0.186 \\
\hline Employed & 0.20 & 1.74 & 0.01 & -3.22 & 3.61 & 0.909 \\
\hline Retired & -1.03 & 1.64 & -0.05 & -4.26 & 2.20 & 0.530 \\
\hline Health insurance & 5.60 & 4.15 & 0.06 & -2.56 & 13.76 & 0.178 \\
\hline $\begin{array}{c}\text { Spanish Language } \\
\text { (1-4) }\end{array}$ & -0.60 & 0.57 & -0.07 & -1.71 & 0.52 & 0.294 \\
\hline Acculturation & -0.09 & 0.06 & -0.10 & -0.22 & 0.03 & 0.146 \\
\hline Latino Identity & 0.01 & 0.71 & 0.00 & -1.37 & 1.40 & 0.983 \\
\hline SRH (1-5) & 1.40 & 0.40 & 0.19 & 0.62 & 2.19 & 0.000 \\
\hline ADL & 0.67 & 0.36 & 0.09 & -0.04 & 1.38 & 0.064 \\
\hline CMC (n) & 0.42 & 0.28 & 0.08 & -0.14 & 0.98 & 0.139 \\
\hline Smoking (Current) & 0.70 & 1.16 & 0.03 & -1.57 & 2.98 & 0.543 \\
\hline Polypharmacy & 2.54 & 1.06 & 0.12 & 0.45 & 4.62 & 0.017 \\
\hline Intercept & 6.40 & 8.42 & & -10.15 & 22.95 & 0.447 \\
\hline
\end{tabular}

SRH: Self-Rated Health. ADLs: Activities of Daily Living. CMCs: Chronic Medical Conditions.

In Model 1, gender was associated with depressive symptoms, with men reporting lower depressive symptoms than women. In Model 2, which also included SES indicators, retirement, and health insurance, high education and income were associated with less depressive symptoms. In Model 3, no cultural variables were associated with depressive symptoms. In Model 4, all health variables, including SRH, ADL, and CMCs, were positively associated with depressive symptoms. In Model 5, smoking did not correlate with depressive symptoms. In Model 6 polypharmacy was associated with depressive symptoms, above and beyond all the covariates.

\section{Discussion}

Using data from the SALSA-2008 study, the current study showed that polypharmacy is associated with depressive symptoms in U.S.-born older Mexican Americans. This association was independent of all confounders including demographic factors, SES, retirement, health insurance, acculturation, language proficiency, smoking, physical health, and health behaviors.

Previous studies have shown an association between polypharmacy and poor mental health (e.g., clinical depression and psychological distress) in other ethnic groups [6,7]. These studies have been unable to determine if the diagnosis of depression and antidepressant pharmacotherapeutic agents directly increase the risk of polypharmacy, or if polypharmacy deteriorates mental health 
through drug-drug interactions [6,7]. Thus, more research is needed on the direction of the effect between polypharmacy and depressive symptoms in ethnic older adults.

Inappropriate polypharmacy should be prevented in Latino older Americans, and this may contribute to mental health promotion in this population. Several strategies are proposed to reduce inappropriate polypharmacy in older adults [40]. Deprescribing is considered one of the most evidence-based approaches for reducing inappropriate polypharmacy [40]. Recommended protocols include spending more time reviewing patients' medications. It has been demonstrated that even spending 10 minutes to review elderly patients' medications can reduce the rate of polypharmacy [41]. Deprescribing has promising effects on increasing medication adherence, by simply reducing the number of medications taken [40]. These efforts can be applied by physicians, nurses [40], or pharmacists [42]. Mnemonics, algorithms, clinical practice guidelines, and clinical strategies have proposed tools to address polypharmacy in a variety of health care settings [43]. Furthermore, a systematic review of the strategies to reduce psychiatric polypharmacy demonstrated that modest interventions that utilize educational approaches, as well as more assertive interventions that directly caution physicians to avoid polypharmacy, have both been effective in reducing polypharmacy [44].

More research is needed on the most effective ways by which we can reduce inappropriate polypharmacy, particularly in ethnic minority groups. This includes tailored interventions that are targeted specifically to each cultural group. The present literature has several limitations including lack of adequate control variables, low sample size, poor measurement, lack of access to medications taken by participants, duration of follow up, poor randomization, and inadequate monitoring of potential adverse effects. More research is needed to overcome these methodological limitations [45]. More research is also needed to understand how we can reduce inappropriate polypharmacy across ethnic groups including Mexican Americans. Very few studies have tested the role of tailored interventions for this specific population.

We went beyond exploring social determinants of polypharmacy $[3,18]$, and suggested that depressive symptoms may be a consequence of polypharmacy in Mexican Americans. As polypharmacy is more commonly seen in individuals with low educational attainment and income $[3,46]$, ethnic minorities with low SES should be a key target population for program and intervention planning to prevent inappropriate polypharmacy $[3,18]$.

Unfortunately, prevalence of polypharmacy is particularly increasing in low SES individuals [4]. Very few studies have been conducted on trends of polypharmacy in Latino and Mexican Americans based on country of origin, immigration, nativity, and culture. Thus, there is a need to conduct more research on this population.

This study focused on older Mexican Americans in Sacramento county. The same results may not apply to other ethnic groups including older White and African Americans who reside in Sacramento county, or even Mexicans who were born and reside in other geographical areas within the United States. As such we cannot simply assume that what is relevant to U.S.-born older Mexican Americans would apply to other groups of Mexicans. Thus, more tailored and intersectional research is needed that considers country of origin, ethnicity, and immigration status. These social factors shape available resources, vulnerabilities, values, health needs, mindset, health care use, and response to health care need.

Severity of depressive symptoms, which reflects a clinical diagnosis of depression, can directly increase the risk of polypharmacy, particularly in individuals with multiple chronic conditions. Individuals with clinical depression, severe depression, and suicidality may receive medications specifically to treat depression. Similarly, treatment resistant depression may increase the chance of receiving multiple medications. This study, however, did not assess the type of medications that were prescribed. The complex interplay between antidepressant pharmacotherapeutic agents and other medications requires more research [47].

This study was a quantitative rather than a qualitative analysis of polypharmacy and depressive symptomatology. Future research may assess the exact types of medication that are being taken by the 
included participants. We do not know which percentage of the sample receive psychopharmacotherapy or what medications were most frequently prescribed in the sample. The lack of such information in the current analysis requires additional research.

\section{Limitations}

No study is without limitations. One limitation of this study was its cross-sectional design. While depression predicts polypharmacy, polypharmacy may also impact mental health. In addition, SES and health behaviors may also impact both polypharmacy and mental health. Thus, we cannot draw any causal conclusion from our observation of a cross-sectional association between depressive symptoms and polypharmacy. Another limitation was that we only included U.S.-born older Mexican Americans. Other ethnic groups, immigrants, and Latinos of differing nativity, such as Puerto Ricans and Cubans, should also be included in future research regarding depressive symptoms and polypharmacy. We used a self-rating scale of depressive symptoms. The results from these self-rating scales may differ from those standardized rating scales such as the Hamilton depression measure.

We still do not know why older people who are using five or more medications a day should have higher depressive symptoms. We did not exclude subjects who used psychiatric medications, however, as participants' full psychiatric and medication histories were not assessed. The type of medications, as well as components of polypharmacy, may differ in individuals with and without depression and other psychiatric disorders. One hypothesis is that more prescriptions may reflect the presence of clinical depression from several psychiatric and/or chronic comorbidities that have resulted in polypharmacy. We also did not know for how long the subjects had been taking their medications prior to the assessment of their polypharmacy or depressive symptoms. Despite these limitations, this study still makes a contribution by extending the existing knowledge on mental health correlates of polypharmacy in ethnic minority groups in the U.S.

\section{Conclusions}

Polypharmacy is linked to high depressive symptoms among U.S.-born older Mexican Americans. This link is independent of demographic factors, SES, health behaviors, physical health, healthcare access, acculturation, and language proficiency. More research is needed on interceptions that can most effectively reduce inappropriate polypharmacy among older Mexican Americans.

Author Contributions: Conceptualization, S.A.; Data curation, S.A.; Formal analysis, S.A.; Funding acquisition, M.B.; Writing—original draft, S.A. and C.W.; Major revision, C.W.; Writing—review and editing, all authors.

Funding: Bazargan and Assari are supported by the following National Institutes of Health (NIH) grants: D084526-03, CA201415 02, DA035811-05, U54MD008149, U54MD007598, and U54CA229974 and a Center for Medicare and Medicaid Services (CMS) Grant 1H0CMS331621. Assari was also supported by the National Institute of Minority Health and Health Disparities (NIMHD) award 5S21MD000103.

Conflicts of Interest: The authors declare no conflict of interests.

\section{References}

1. Leelakanok, N.; Holcombe, A.L.; Lund, B.C.; Gu, X.; Schweizer, M.L. Association between polypharmacy and death: A systematic review and meta-analysis. J. Am. Pharm. Assoc. 2017, 57, 729-738. [CrossRef]

2. Armstrong, K.S.; Temmingh, H. Prevalence of and factors associated with antipsychotic polypharmacy in patients with serious mental illness: Findings from a cross-sectional study in an upper-middle-income country. Rev. Bras. de Psiquiatr. 2017, 39, 293-301. [CrossRef]

3. Assari, S.; Bazargan, M. Race/Ethnicity, Socioeconomic Status, and Polypharmacy among Older Americans. Pharmacy 2019, 7, 41. [CrossRef]

4. Baek, Y.-H.; Shin, J.-Y. Trends in polypharmacy over 12 years and changes in its social gradients in South Korea. PLoS ONE 2018, 13, e0204018. [CrossRef] [PubMed] 
5. Haider, S.I.; Johnell, K.; Thorslund, M.; Fastbom, J. Analysis of the association between polypharmacy and socioeconomic position among elderly aged $\geq 77$ years in Sweden. Clin. Ther. 2008, 30, 419-427. [CrossRef] [PubMed]

6. Assari, S.; Bazargan, M. Polypharmacy and Psychological Distress May Be Associated in African American Adults. Pharmacy 2019, 7, 14. [CrossRef] [PubMed]

7. Bazargan, M.; Smith, J.; Saqib, M.; Helmi, H.; Assari, S. Associations between Polypharmacy, Self-Rated Health, and Depression in African American Older Adults; Mediators and Moderators. Int. J. Environ. Res. Public Health 2019, 16, 1574. [CrossRef]

8. Trenaman, S.C.; Rideout, M.; Andrew, M.K. Sex and gender differences in polypharmacy in persons with dementia: A scoping review. SAGE Open Med. 2019, 7, 2050312119845715. [CrossRef]

9. Perry, B.A.; Turner, L.W. A Prediction Model for Polypharmacy: Are Older, Educated Women More Susceptible to an Adverse Drug Event? J. Women Aging 2001, 13, 39-51. [CrossRef]

10. Assari, S.; Wisseh, C.; Bazargan, M. Obesity and Polypharmacy among African American Older Adults: Gender as the Moderator and Multimorbidity as the Mediator. Int. J. Environ. Res. Public Health 2019, 16, 2181. [CrossRef]

11. Savoie, I.; Morettin, D.; Green, C.J.; Kazanjian, A. Systematic review of the role of gender as a health determinant of hospitalization for depression. Int. J. Technol. Assess. Health Care 2004, 20, 115-127. [CrossRef] [PubMed]

12. Abate, K.H. Gender disparity in prevalence of depression among patient population: a systematic review. Ethiop. J. Health Sci. 2013, 23, 283-288. [CrossRef] [PubMed]

13. Piccinelli, M.; Wilkinson, G. Gender differences in depression. Br. J. Psychiatry 2000, 177, 486-492. [CrossRef] [PubMed]

14. Culbertson, F.M. Depression and gender: An international review. Am. Psychol. 1997, 52, 25-31. [CrossRef]

15. Luppa, M.; Sikorski, C.; Luck, T.; Ehreke, L.; Konnopka, A.; Wiese, B.; Weyerer, S.; König, H.-H.; Riedel-Heller, S. Age- and gender-specific prevalence of depression in latest-life-Systematic review and meta-analysis. J. Affect. Disord. 2012, 136, 212-221. [CrossRef]

16. Gilman, S.E. Review: There is marked socioeconomic inequality in persistent depression. Évid. Based Ment. Health 2003, 6, 75. [CrossRef]

17. Richardson, R.; Westley, T.; Garièpy, G.; Austin, N.; Nandi, A. Neighborhood socioeconomic conditions and depression: a systematic review and meta-analysis. Soc. Psychiatry Psychiatr. Epidemiol. 2015, 50, 1641-1656. [CrossRef]

18. Assari, S.; Helmi, H.; Bazargan, M. Polypharmacy in African American Adults: A National Epidemiological Study. Pharmacy 2019, 7,33. [CrossRef]

19. Muscat, P.; Chilcot, J.; Weinman, J.; Hudson, J. Exploring the relationship between illness perceptions and depression in patients with chronic kidney disease: A systematic literature review. J. Ren. Care 2018, 44, 174-185. [CrossRef]

20. Kok, G.D.; Bockting, C.L.H.; Burger, H.; Hannig, W.; Pijnenborg, G.H.M.; Cuijpers, P.; Hollon, S.D. Double Trouble: Does Co-Morbid Chronic Somatic Illness Increase Risk for Recurrence in Depression? A Systematic Review. PLoS ONE 2013, 8, e57510. [CrossRef]

21. Clarke, D.M.; Currie, K.C. Depression, anxiety and their relationship with chronic diseases: A review of the epidemiology, risk and treatment evidence. Med. J. Aust. 2009, 190, S54-S60. [CrossRef] [PubMed]

22. Assari, S.; Lankarani, M.M. Does Multi-morbidity Mediate the Effect of Socioeconomics on Self-rated Health? Cross-country Differences. Int. J. Prev. Med. 2015, 6, 85. [CrossRef] [PubMed]

23. Assari, S. Cross-country variation in additive effects of socio-economics, health behaviors, and comorbidities on subjective health of patients with diabetes. J. Diabetes Metab. Disord. 2014, 13, 36. [CrossRef] [PubMed]

24. Assari, S.; Lankarani, R.M.; Lankarani, M.M. Cross-country differences in the association between diabetes and disability. J. Diabetes Metab. Disord. 2014, 13, 3. [CrossRef] [PubMed]

25. Assari, S. Cross-Country Differences in the Additive Effects of Socioeconomics, Health Behaviors and Medical Comorbidities on Disability among Older Adults with Heart Disease. J. Tehran Univ. Hear. Cent. 2015, 10, 24-33.

26. Assari, S. Combined Racial and Gender Differences in the Long-Term Predictive Role of Education on Depressive Symptoms and Chronic Medical Conditions. J. Racial Ethn. Health Disparities 2017, 4, 385-396. [CrossRef] 
27. Garcia, L.; Lee, A.; Al Hazzouri, A.Z.; Neuhaus, J.; Epstein, M.; Haan, M. The Impact of Neighborhood Socioeconomic Position on Prevalence of Diabetes and Prediabetes in Older Latinos: The Sacramento Area Latino Study on Aging. Hisp. Health Care Int. 2015, 13, 77-85. [CrossRef]

28. Rodriquez, E.J.; Sabado-Liwag, M.; Perez-Stable, E.J.; Lee, A.; Haan, M.N.; Gregorich, S.E.; Jackson, J.S.; Napoles, A.M. Allostatic Load, Unhealthy Behaviors, and Depressive Symptoms by Birthplace Among Older Adults in the Sacramento Area Latino Study on Aging (SALSA). J. Aging Health 2019. [CrossRef]

29. Al Hazzouri, A.Z.; Haan, M.N.; Osypuk, T.; Abdou, C.; Hinton, L.; Aiello, A.E. Neighborhood Socioeconomic Context and Cognitive Decline Among Older Mexican Americans: Results from the Sacramento Area Latino Study on Aging. Am. J. Epidemiol. 2011, 174, 423-431. [CrossRef]

30. Haan, M.N.; Al-Hazzouri, A.Z.; Aiello, A.E. Life-span Socioeconomic Trajectory, Nativity, and Cognitive Aging in Mexican Americans: The Sacramento Area Latino Study on Aging. J. Gerontol. Ser. B 2011, 66, i102-i110. [CrossRef]

31. Al Hazzouri, A.Z.; Haan, M.N.; Kalbfleisch, J.D.; Galea, S.; Lisabeth, L.D.; Aiello, A.E. Life-Course Socioeconomic Position and Incidence of Dementia and Cognitive Impairment Without Dementia in Older Mexican Americans: Results from the Sacramento Area Latino Study on Aging. Am. J. Epidemiol. 2011, 173, 1148-1158. [CrossRef] [PubMed]

32. Afable-Munsuz, A.; Mayeda, E.R.; Pérez-Stable, E.J.; Haan, M.N. Immigrant Generation and Diabetes Risk Among Mexican Americans: The Sacramento Area Latino Study on Aging. Am. J. Public Health 2014, 104, S243-S250. [CrossRef] [PubMed]

33. Gonzalez, H.M.; Haan, M.N.; Hinton, L. Acculturation and the prevalence of depression in older Mexican Americans: baseline results of the Sacramento Area Latino Study on Aging. J. Am. Geriatr. Soc. 2001, 49, 948-953. [CrossRef] [PubMed]

34. Radloff, L.S. The CES-D scale: A self-report depression scale for research in the general population. Appl. Psychol. Meas. 1977, 1, 385-401. [CrossRef]

35. Katz, S.; Ford, A.B.; Jackson, B.A.; Jaffe, M.W.; Moskowitz, R.W. Studies of Illness in the Aged: The Index of ADL: A Standardized Measure of Biological and Psychosocial Function. JAMA 1963, 185, 914. [CrossRef] [PubMed]

36. Lawton, M.P.; Brody, E.M. Assessment of Older People: Self-Maintaining and Instrumental Activities of Daily Living. Gerontologist 1969, 9, 179-186. [CrossRef]

37. Jadalla, A.; Lee, J. Validation of Arabic and English versions of the ARSMA-II Acculturation Rating Scale. J. Immigr. Minor. Health 2015, 17, 208-216. [CrossRef]

38. Jimenez, D.E.; Gray, H.L.; Cucciare, M.; Kumbhani, S.; Gallagher-Thompson, D. Using the Revised Acculturation Rating Scale for Mexican Americans (ARSMA-II) with Older Adults. Hisp. Health Care Int. 2010, 8, 14-22. [CrossRef]

39. Martinez, C.R.; Schwartz, S.J.; Thier, M.; McClure, H.H. A tale of two measures: Concordance between the ARSMA-II and the BIQ acculturation scales among Latino immigrant families. Psychol. Assess. 2018, 30, 459-473. [CrossRef]

40. Ulley, J.; Harrop, D.; Ali, A.; Alton, S.; Davis, S.F. Deprescribing interventions and their impact on medication adherence in community-dwelling older adults with polypharmacy: A systematic review. BMC Geriatr. 2019, 19, 15. [CrossRef]

41. Walsh, E.K.; Cussen, K. “Take ten minutes": A dedicated ten minute medication review reduces polypharmacy in the elderly. Ir. Med. J. 2010, 103, 236-238. [PubMed]

42. Van Der Meer, H.G.; Wouters, H.; Pont, L.G.; Taxis, K. Reducing the anticholinergic and sedative load in older patients on polypharmacy by pharmacist-led medication review: A randomised controlled trial. BMJ Open 2018, 8, e019042. [CrossRef] [PubMed]

43. Skinner, M. A literature review: Polypharmacy protocol for primary care. Geriatr. Nurs. 2015, 36, 367-371. [CrossRef] [PubMed]

44. Tani, H.; Uchida, H.; Suzuki, T.; Fujii, Y.; Mimura, M. Interventions to reduce antipsychotic polypharmacy: A systematic review. Schizophr. Res. 2013, 143, 215-220. [CrossRef] [PubMed]

45. Tranulis, C.; Skalli, L.; LaLonde, P.; Nicole, L.; Stip, E. Benefits and risks of antipsychotic polypharmacy: An evidence-based review of the literature. Drug Saf. 2008, 31, 7-20. [CrossRef] 
46. Xiang, Y.-T.; Weng, Y.-Z.; Leung, C.-M.; Tang, W.-K.; Ungvari, G.S. Clinical and Social Determinants of Antipsychotic Polypharmacy for Chinese Patients with Schizophrenia. Pharmacopsychiatry 2007, 40, 47-52. [CrossRef]

47. Dold, M.; Bartova, L.; Mendlewicz, J.; Souery, D.; Serretti, A.; Porcelli, S.; Zohar, J.; Montgomery, S.; Kasper, S. Clinical correlates of augmentation/combination treatment strategies in major depressive disorder. Acta Psychiatr. Scand. 2018, 137, 401-412. [CrossRef]

(c)

(C) 2019 by the authors. Licensee MDPI, Basel, Switzerland. This article is an open access article distributed under the terms and conditions of the Creative Commons Attribution (CC BY) license (http://creativecommons.org/licenses/by/4.0/). 